\title{
Association of high cortisol levels in pregnancy and poor postpartum mental health and altered fetal growth. Analysis in the MAASTHI cohort study, Bengaluru
}

\section{Giridhara R Babu ( $\nabla$ epigiridhar@gmail.com )}

Indian Institute of Public Health, Public Health Foundation of India

\section{Prafulla Shriyan}

Indian Institute of Public Health, Public Health Foundation of India

\section{Paulomi Sudhir}

National Institute of Mental Health and Neurosciences

\section{Onno CP Schayck}

Maastricht University

\section{Research Article}

Keywords: Pregnancy, serum cortisol, fetal growth, stress, biomarker, anthropometry

Posted Date: January 27th, 2022

DOI: https://doi.org/10.21203/rs.3.rs-1150688/v1

License: (c) (1) This work is licensed under a Creative Commons Attribution 4.0 International License.

Read Full License 


\title{
Association of high cortisol levels in pregnancy and poor postpartum mental health and altered fetal growth.
}

\section{Analysis in the MAASTHI cohort study, Bengaluru.}

\author{
Prafulla Shriyan ${ }^{1}$, Paulomi Sudhir², Onno CP van Schayck ${ }^{3}$, Giridhara R Babu ${ }^{1,4}$
}

1. Indian Institute of Public Health, Public Health Foundation of India, Bengaluru, Karnataka, 560023, India

2. Clinical Psychology, National Institute of Mental Health and Neurosciences.

3. Care and Public Health Research Institute, Maastricht University, Maastricht, Limburg, The Netherlands

4. The Wellcome Trust/DBT India Alliance, New Delhi, 110025, India

\section{Corresponding author: epigiridhar@gmail.com}

\section{MBBS, MPH, PhD (UCLA). Professor and Head, Life course Epidemiology, IIPH Bangalore, Public Health Foundation of India.}

\begin{abstract}
:
Background: The role of maternal stress levels on mothers' mental health and fetal growth has been previously studied. We aim to investigate the association of serum cortisol level in pregnancy with birth outcomes and postpartum depressive symptoms in a public health facility, India.

Methods: The current study is a part of the MAASTHI prospective cohort that assessed the relationship between maternal psychosocial environment and adverse neonatal outcomes and maternal mental health after birth. We assessed serum cortisol levels in stored blood samples in 230 pregnant women as a biomarker for stress during pregnancy. Pregnant women between 18 and 45 years of age, presenting at $\geq 14$ weeks of gestation and providing voluntary written informed consent were recruited in the study. The Edinburgh Postnatal Depression Scale was used to assess postpartum depressive symptoms and detailed infant anthropometric measurements were carried out at birth.
\end{abstract}

Results: A serum cortisol threshold value of $>17.60 \mathrm{IU} / \mathrm{L}$ seemed to be associated with postpartum depressive symptoms, low birth weight, and lower weight for length/height z-score. No significant association was found between serum cortisol and infants reduced head circumference, the sum of skinfold thickness, and crown-rump length.

Conclusion: Our results support the hypothesis that higher maternal cortisol levels may adversely affect a mother's mental health and birth outcomes. These findings have implications for understanding maternal mental health and early developmental psychopathology, both of which are well-known but understudied. Future studies should explore this association at various gestational ages and use epigenetic markers to determine hypothalamic-pituitary axis (HPA) activity during pregnancy and its effect on birth outcomes.

Keywords: Pregnancy, serum cortisol, fetal growth, stress, biomarker, anthropometry. 


\section{Background:}

Pregnancy induces significant changes in the levels and function of the endocrine systems; these changes affect the mother's mental health (1) and fetal development (2). Women may experience stress in the form of anxiety, depression, social isolation, and pathological conditions. Pregnancy-specific stress may be in any form, including worries about prenatal screening results, concern about the health and development of the baby, and ambiguity about the life changes associated with motherhood. (3) Stress during pregnancy is harmful to the health of the mother and the fetus. Recent evidence indicates that it may have long-term consequences (4) related to growth retardation, organ maturation, attachment difficulties, infant motor and cognitive development, and decreased intelligence quotient $(\mathrm{IQ}) \cdot(4,5)$

Fetal development ensures the optimal health of the child in future. Birth outcomes are influenced by factors such as diet, prenatal smoking, gestational diabetes, and hypertension.(6) Mental health status during pregnancy significantly impacts the onset and persistence of depression. Also, periconceptional and postpartum psychological factors influence fetal growth development.(7) Previous studies have shown that elevated psychosocial stress in pregnancy increases the risk of poor birth outcomes (8) and postpartum depressive symptoms.(9) Poor maternal mental health due to depression in pregnancy may lead to social isolation(10), marital conflicts(11), delays in child motor or intellectual development(12), lower fetal growth(13), and greater stress reactivity in children.(13) Infants born to women suffering from postpartum depressive symptoms are more likely to develop mental health problems later in life. (14) Several mechanisms have been hypothesized to explain elevated cortisol levels during pregnancy on maternal mental health and child development.

Cortisol levels are linked to fetal growth and mental health difficulties in mothers.(15) Cortisol is a stress hormone released in response to stress and is a key marker for the activation of the stress response.(16) The Hypothalamicpituitary-adrenal axis (HPA) system regulates cortisol,(17) with the placenta releasing cortisol and contributing to the higher cortisol levels in pregnant women. The high secretion of cortisol (hypercortisolemia) reduces the stress response and inhibits hypothalamic activity.(18) The probability of developing postpartum depressive symptoms increases when an external stressor is involved.(19) When a fetus is exposed to cortisol in the womb, the enzyme 11-hydroxysteroid dehydrogenase type 2 (11-HSD2) converts cortisol to its inactive form cortisone. Stress stimulates cortisol secretion due to a drop in placental enzymatic activity(11-HSD2).(20) This causes uterine artery vasoconstriction, restricts blood supply to the fetus, and results in poor birth outcomes.(21) 
Studies during pregnancy investigating the effects of the HPA on birth outcomes and maternal mental health status have shown mixed results. Among the few studies that have assessed the relationship between serum cortisol and birth outcomes, most were assessed in a small sample size (between 6 and 98).(22, 23) Studies that could not establish an association between cortisol and birth outcomes suffered from an inadequate sample size. $(24,25)$ Results of studies exploring a relationship between cortisol and postpartum depressive symptoms were inconclusive and have shown both hypercortisolemia and hypocortisolemia to be associated with postpartum depressive symptoms.(9) This difference in results may be due to differences in methodology, insufficient sample size, type of biological samples assessed, nature of stress responses in different populations, interpretation of cortisol levels, use of self-report measures to screen postpartum depressive symptoms, and circadian nature of the hormone. Previous studies have obtained information on stress during pregnancy via interviews with mothers. Standardized questionnaires, when used alone, have limitations in assessing depression as they only reflect perspectives that change during pregnancy.(26) Hence, they need to be combined with biomarker assessments in objectively identifying those at risk of stress.(27) To our knowledge, there is no current evidence from lower- and middleincome countries (LMICS) that illustrates an association between cortisol level during pregnancy on both infants physical growth outcomes and mother's mental health status using cohort-based samples.

We aimed to understand the association of elevated serum cortisol during pregnancy on birth outcomes and postpartum depressive symptoms. We hypothesized that fetal exposure to higher pregnancy cortisol levels would be linked with a higher risk of altered growth parameters in children and a higher risk of developing postpartum depressive symptoms in mothers assessed after delivery. Altered growth parameters included birth weight, small weight-for-gestational age, weight for length/height, and head circumference.

\section{Methods}

Design: The study participants were part of the 'Maternal antecedents of adiposity and studying the transgenerational role of hyperglycemia and insulin' (MAASTHI) cohort. This prospective cohort study began in 2016. The objective of the cohort study was to assess the association between the nutritional and psychosocial environment in pregnancy with adverse neonatal outcomes, including adiposity. The MAASTHI cohort consisted of 4862 pregnant women with a mean age of 24.25 years (SD \pm 4.06 ). Baseline questionnaire assessment was conducted among eligible participants who were $>14$ weeks of gestation, aged between 18 and 45 years, and resided in the study location for five years. Details of the overall study design, methods, and outcomes have been previously 
published elsewhere.(28) For the present study, a serum cortisol assessment was conducted among a sub-sample of pregnant women who had completed 24 weeks of gestation. We estimated the sample size using $n$ $=\left(Z_{\alpha} / 2+Z_{\beta}\right) 2 p q / d^{2}$. The " $p$ " was obtained from the results of the cohort, in which the prevalence of small for gestational age infants among the pregnant women who had depressive symptoms was $21.3 \%,(29)$; along with a power of $80 \%$, a $95 \%$ confidence interval and a $10 \%$ precision, the computed sample size was 91 . After accounting for a $60 \%$ non-response rate, 230 women were included.

\section{Data collection}

Demographic and obstetric characteristics: At enrollment, trained research staff obtained sociodemographic details, obstetric history, depression status using Edinburgh Postnatal Depression Scale (EPDS), social support and detailed anthropometric measurements from the participants. Additionally, participants' blood haemoglobin and gestational diabetes status were assessed, and blood samples were stored for advanced analysis. Obstetric information on gravida, parity, history of abortion and stillbirth, morbidities during pregnancy, gestational diabetes, and hypertension was obtained from each participant. From the stored samples, 230 samples were taken from the 'Biorepository' for cortisol estimation. Samples from the biorepository were shipped to MEDALL Healthcare Private Ltd., which is a nationally accredited laboratory. MEDALL Healthcare Private Ltd. has been certified by the International Organization for Standardization (ISO) and the National Accreditation Board for testing and calibration Laboratories (NABL).

\section{Cortisol assessment}

A trained phlebotomist performed a venipuncture to collect a six $\mathrm{ml}$ blood sample from each participant for serum preparation. The vacutainer was wrapped in aluminium foil to protect it from sunlight and kept for 45 minutes for coagulation. It was subsequently centrifuged for 10 minutes at $1500 \mathrm{rpm} /$ minute. The clear supernatant serum was transferred into freeze-resistant black coloured aliquots. The analytical work was conducted in a specialized laboratory. These freeze-dried samples were subsequently shipped in an insulated container that was adequately filled with dry ice to laboratories for conducting assays. Using an electrochemiluminescence immune assay method, a fully automated analyzer determined serum cortisol concentration, reported in ug/L. Cortisol concentration was measured using an enzyme-linked immunosorbent assay (ELISA) method. The lower and upper limits of measurements were $2.16 \mathrm{ug} / \mathrm{L}$ and $32.86 \mathrm{ug} / \mathrm{dl}$, respectively. 


\section{Maternal psychosocial environment assessment}

Depressive symptoms were assessed using the Edinburgh Postnatal Depression Scale (EPDS). EPDS has been extensively used to screen depression during pregnancy and postpartum periods. It uses a 10 -item scale, with each item having a score from 0 to 3 and has a maximum score of 30. A cut-off score of 11 or 12 on the EPDS detected depression with a specificity of $85 \%$ and a sensitivity of $92 \%$.(30) For this study, we considered a score of $0-12$ to indicate 'no depressive symptoms, and a score of 13 or higher to indicate 'clinically-relevant depressive symptoms'. A questionnaire was used to measure social support, including emotional, instrumental, informational, and appraisal components. This questionnaire comprised a 12-item scale, with each item having a score from 0 to 3 and a maximum score of 36 . A cut-off score of 24 was considered to classify the scores into "good" social support and "poor" social support.(31)

\section{Outcomes}

1. Maternal postpartum depressive symptoms: The EPDS tool has been used to assess the postpartum depressive symptoms after birth within 72 hours of delivery.

2. Birth outcomes: The birth outcome variables were:

- low birth weight (Yes/No); low birth weight was defined as birth weight less than 2.5 kilograms.

- Small for gestational age (Yes/No); small for gestational age (SGA) was defined as birth weight below $10^{\text {th }}$ percentile for gestational age based on gender.

- Lower weight for height/length (Yes/No); lower weight for length/height was defined as WHZ scores below the $10^{\text {th }}$ percentile for gestational weight based on gender.

- Lower head circumference (Yes/No); lower head circumference was defined as HC Z-scores below $10^{\text {th }}$

percentile for gestational age based on gender.

- Lower skinfold thickness (Yes/ No); lower skinfold thickness was defined as the sum of skinfold thickness below the $10^{\text {th }}$ percentile.

- Lower crown-rump length (Yes/No); lower crown-rump length (CRL) was defined as CRL below the $10^{\text {th }}$

percentile.

The enrolled pregnant women were followed from pregnancy to childbirth. The infants' age, gender, weight, length, circumferences, and skinfold thickness were assessed. The infant's weight was recorded to the nearest 
10 grams using a SECA 381 baby weighing scale. The infant's length was recorded to the nearest 1 centimetre using a SECA Infantometer. Skinfold thickness was measured using a Holtan calliper (UK). Circumferences were measured using a Chasmors body circumference tape. Three readings were taken for every anthropometric measurement.

\section{Covariates}

Based on a priori, we included the following covariates: gestational age at testing, obstetric morbidity, age, education, socioeconomic status, gravida, parity, gestational diabetes and hypertension, body mass index, history of previous abortion, social support, depressive symptoms during pregnancy, gestational age at delivery, type of delivery and gender of the child. Information on these covariates was obtained from participants during enrollment and follow-up assessment. Confounding variables were maternal age, socioeconomic status, spousal smoking and alcohol habit, maternal education, gestational age at testing, gravida, parity, body mass index (BMI), gestational diabetes, hypertension, depressive symptoms during pregnancy, social support, gestational age at delivery, type of delivery and infant gender.

\section{Statistical analysis}

Maternal cortisol levels were included in the analysis in two different ways: 1) continuous and 2) categorical. Cortisol levels were trichotomized as low $\left(<25^{\text {th }}\right.$ percentile), moderate $\left(25^{\text {th }}-75^{\text {th }}\right.$ percentile), and high $\left(>75^{\text {th }}\right.$ percentile), and dichotomized as normal and high by the mean cut off level (mean cortisol=17.66 ug/L). Linear regression analysis was performed to examine the independent association between serum cortisol and infant outcomes by initially adjusting for gestational age, infant gender, maternal age, parity, BMI, and gestational age at delivery. After adjusting for potential confounders, we also performed a logistic regression analysis to find the association between maternal cortisol threshold (percentile cut-off \& mean cut-off) with infant outcomes.

A similar set of analyses was carried out for maternal cortisol levels to evaluate its association with maternal postpartum depressive symptoms, including both continuous and categorical variables of serum cortisol adjusting for age, socioeconomic status, maternal education, gravida, H/o abortion, BMI, gestational diabetes, hypertension, depressive symptoms during pregnancy, social support, gestational age at delivery, type of delivery and the gender of the child. We present the correlation coefficients, the odds ratio (wherever applicable), 95\% confidence interval, 
and p-value. All analyses were performed using the Statistical Package for the Social Science (SPSS) version 23 statistical software.(32)

\section{Results}

Baseline maternal characteristics of the participants are displayed in Table 1. Most of the participants were above 30 years of age $(65.7 \%)$, practiced Islam religion $(52.6 \%)$, followed by participants who identified themselves as Hindus (43.5\%). A relative majority had a high school education (42\%). Nearly $65 \%$ of the participants belonged to an upper-class family; $37.5 \%$ reported smoking habits among their spouses. Nearly $18 \%$ of them were found to have depressive symptoms during the current pregnancy.

Table 1. Maternal and infant characteristics and their association with maternal cortisol levels $(n=230)$

We found a statistically significant difference for the following variables; maternal age, gravida, infant's gender and infant's weight for height $z$-score below $10^{\text {th }}$ percentile in women with higher levels compared with normal cortisol levels. (Table-1)

Further, the results of the linear regression analysis (Table-2) indicate that maternal cortisol levels was not associated with birth weight $(b=0.015 ; p$-value $\geq 0.05)$, SGA $(-0.010, p$-value $>0.05)$, lower weight for length/height (0.009, $p$-value $>0.05)$ and lower head circumference $(0.006, p$-value $>0.05)$. Based on the three categories of cortisol levels low $\left(<25^{\text {th }}\right.$ percentile), moderate $\left(25^{\text {th }}-75^{\text {th }}\right.$ percentile), high $\left(>75^{\text {th }}\right.$ percentile), we did not find a significant association between moderate or high cortisol levels and low birth weight, SGA, lower weight for length/height z-score, lower skinfold thickness, lower head circumference and lower crown-rump length. However, after adjusting for potential confounders, cortisol levels, when categorized using a mean cut-off of $17.66 \mathrm{ug} / \mathrm{L}$, showed a statistically significant association between low birth weight (OR=2.28; $95 \% \mathrm{Cl} 1.21$ to 4.32 ) and lower weight for length/height ( $\mathrm{OR}=2.16 ; 95 \% \mathrm{Cl} 1.07$ to 4.35). Pregnant women with cortisol levels above 17.66 $\mathrm{ug} / \mathrm{L}$ had twice the odds of delivering a baby with low birth weight and lower weight for length/height compared to those with cortisol levels below the mean cut-off level. We did not find a significant association for other outcome measures such as SGA, lower head circumference, lower skinfold thickness, and lower crown-rump length. 
Table 2. Regression results on the association between maternal cortisol level and birth weight, small for gestational age (SGA), length for gestational weight, and head circumference for gestational age, $n=230$

Table 3. Logistic regression analysis for elevated cortisol during pregnancy and postpartum depression.

After adjusting for a range of sociodemographic, obstetric, and infant variables, we observed that cortisol levels during pregnancy with a higher mean cut off of $>17.66 \mathrm{ug} / \mathrm{L}$ were substantially associated with postpartum depressive symptoms(Table 3). The adjusted model results show a 2.3-fold increased risk of developing postpartum depression among pregnant mothers with higher mean cortisol cut-off levels than those with cortisol levels lower than the mean cut-off. [AOR:2.339, 95\% $\mathrm{Cl}(1.17,4.64)]$. The findings suggest an increased risk of postpartum depressive symptoms when a set of covariates is controlled in the analysis.

\section{Discussion}

We evaluated the association between maternal cortisol levels in pregnancy's second and third trimesters with poor maternal mental health and birth outcomes. We found that lower weight for length/height and gender appeared to be related to higher serum cortisol levels. Our findings show a significant association between high mean cortisol and postpartum depressive symptoms, suggesting that hypercortisolemia leads to postpartum depressive symptoms. Also, we show that exposure to cortisol during pregnancy is associated with lower birth weight and shorter weight for length/height. The suggested mechanism of elevated cortisol concentration potentially induces vasoconstriction of the uterine artery and thereby reducing the uterine blood flow. Restriction of oxygen and nutrients to the fetus further leads to low birth weight.(33)

The findings of our study on the effect of cortisol on poor birth outcomes are consistent with previous findings. The systematic review and metaanalysis findings revealed a negative correlation between maternal salivary cortisol and birth weight.(34)The results from rural Malawi showed a strong association between pregnancy salivary cortisol levels and birth weight.(35) Additionally, study findings from Germany revealed a significant association between maternal cortisol level and birth weight.(36) A comparable study conducted in China found that increased pregnancy cortisol level was associated with decreased birth weight.(37) Several other studies have reported similar 
results.(38),(39). Our results differ from the studies conducted in the developed countries like Netherlands and California) where maternal cortisol levels in pregnancy had no significant associations with infant birth weight. (40) (41)

Overexposure to cortisol has been associated with reduced fetal growth due to, for instance, fetal tissue proliferation and growth inhibition.(42) Elevated cortisol inhibits placental growth, alters blood flow and impairs fetal growth. This association could be a proxy for hormonal, immunological, or placental-derived regulatory mechanisms for fetal growth. $(43,44)$ Inadequate consumption of food, essential fatty acids, or vitamins like folic acid and vitamin B12 could play a potential role in this link. Higher cortisol level during pregnancy is also associated with lower newborn weight for length. This finding is consistent with the results of multiple studies, including the findings from Germany.(45) The findings from Switzerland similarly reported a significant association between exposure to higher serum cortisol during late pregnancy and a short newborn weight for length.(36) A study conducted among a cohort of Singaporean pregnant women found an association between depression and anxiety and low birth weight for length.(46) Our findings differ from the rural Malawi trial, which measured salivary cortisol level but found no association between cortisol level during pregnancy and size at birth.(47) A possible explanation for these observed differences is due to variations in measurement timing and different pools of samples from which cortisol was measured. While we utilized pregnancy serum cortisol concentration, the Malawi trial included salivary cortisol concentrations.

There is no clear evidence in the literature from India and other LMICs on stress biomarkers and their impact on birth outcomes. However, studies that used questionnaires to assess mental health status during pregnancy found a substantial impact on birth outcomes such as low birth weight(48) SGA,(29) and stunting at an early age.(49) Moreover, there is insufficient evidence on the effect of higher cortisol exposure during pregnancy on an infant's skinfold thickness and crown-rump length. This suggests that birth weight and weight for length/height may be affected more due to pregnancy cortisol exposure than other anthropometric markers.

Hormones influence a woman's mental health, foetal growth, and development during pregnancy, and any changes in in-utero maternal hormones can have a significant impact on both the mother and the foetus. As a consequence, 
stress biomarker evaluations should be prioritised. Serum cortisol levels must be assessed at different time points in pregnancy since maternal biochemical profile and physiology vary over time. This study confirms previous findings that biological mechanisms, such as hormonal changes, can significantly impact maternal and infant birth outcomes. In contrast, recent evidence has shown insignificant findings and a negative correlation between cortisol and postpartum depressive symptoms.(50) Our findings imply that hypercortisolemia during pregnancy has a significant impact on maternal mental health after delivery, emphasizing the need for further research on the role of hypercortisolemia in the development of postpartum depression symptoms. So far, most of the studies have focused on the prevalence of postpartum depression and its risk factors in India. Therefore, this study offers evidence for the first time.

The strength of our study includes the use of a prospective study design to assess early morning cortisol assessment during the second or third trimesters of pregnancy. There is reduced variability in the actual cortisol concentration during this time of the day. To control for diurnal variation in the cortisol, we standardized the timing of sample collection during the early morning. Additionally, we controlled the timings by restricting the participants who arrived late. The limitations of this study include small sample size and a one-time assessment of cortisol during pregnancy compared to previous studies that have assessed cortisol concentration during different time points of gestation, i.e. in the first, second and third trimester. On the other hand, non-differential measurement errors could have impacted our study findings.

Pregnancy is a period of transition and adaptation for women as their bodies, thoughts, and emotions change. Thus, an appropriate stress assessment is warranted during this time. In terms of measuring stress, future research should take multiple cortisol measurements over some time throughout pregnancy. Effect on fetal growth has influenced postpartum physiology, metabolism and stress susceptibility, leading to chronic diseases.(51) Given cortisol's capacity to breach the placental barrier,(52) it is necessary to explore the effect of maternal cortisol on fetal programming. These findings will be crucial to determine the role of the HPA axis in fetal growth and development.

\section{Clinical implications}

Our findings revealed that a threshold level of serum cortisol of $>17.60 \mathrm{IU} / \mathrm{L}$ is linked with both maternal mental health and low birth weight and lower weight for length/height z-scores of the infants at birth. This study's findings 10 
illustrate that maternal depression is linked to fetal growth, reflecting an alteration in in-utero programming. These findings could be used to promote awareness of mental health issues and their adverse consequences during pregnancy. They could potentially promote efforts to improve prenatal mental health services in public and private healthcare settings. To minimize the long-term impacts on both the mother and the child, it is necessary to conduct an early stress evaluation of expecting mothers, taking into account stress factors such as maternal age, gravida and the baby's gender preference. The findings will also aid in developing evidence-based solutions, such as stress management programmes, to reduce maternal stress and enhance birth outcomes. Social support and other stress- reduction educational activities should be explored in places where access to mental health is limited.

Conclusion: The results of our study support the hypothesis that maternal cortisol level assessed during pregnancy affects maternal mental health after delivery and fetal birth outcomes such as low birth weight and reduced weight for length/height z-scores of the newborns. These results have significant implications for understanding early developmental psychopathology, which is well-known but poorly understood. Given the challenges of measuring the HPA axis during pregnancy, we focused on a blood biomarker rather than the placenta as a source of potential biomarkers. Future studies should focus on epigenetic markers such as DNA methylation to assess HPA activity during pregnancy. More research is required to study different outcomes about possible mechanisms, maternal physiological parameters and biochemical changes and assess a possible association between stress biomarkers and maternal mental health and birth outcomes.

\section{List of Abbreviations:}

IQ

HPA

11-HSD2

LMICs

MAASTHI

NABL

ELISA

EPDS

LBW

11
Intelligence quotient

Hypothalamic-pituitary-adrenal axis

11-hydroxysteroid dehydrogenase type 2

Lower- and middle-income countries

Maternal antecedents of adiposity and studying the transgenerational role of hyperglycemia and insulin

National Accreditation Board for testing and calibration Laboratories

Enzyme-linked immunosorbent assay

Edinburgh Postnatal Depression Scale

Low birth weight 
$\mathrm{HC}$

Head circumference

CRL

Crown-rump length

BMI

Body mass index

SPSS

Statistical Package for the Social Science

\section{Declaration:}

Ethics approval and consent to participate: The study was conducted according to the guidelines of the Declaration of Helsinki, reviewed and approved by the institutional ethical review board (IEC) at Bangalore campus of Indian Institute of Public Health (Ref No: IIPHHB/TRCIEC/091/2015 Dated 13/11/2015). The study enrolled the participants aged 18 years and above and written informed consent was obtained from all the participants who voluntarily agreed to participate. The study presents anonymous results through publications in journals and reports.

\section{Consent for publication: Not applicable}

Availability of data and materials: The datasets used and analysed during the current study are available from the corresponding author upon request. The data is not publicly available because it contains personal information that could compromise the research participant privacy/consent.

Competing interests: The authors declare that they have no competing interests.

Funding: This work was supported by the Wellcome Trust/DBT India Alliance Fellowship [Grant No. IA/CPHI/14/1/501499] awarded to Giridhara R. Babu. The funding agency had no role in the design and conduct of the study; collection, management, analysis, and interpretation of the data, preparation, review, or approval of the manuscript, or decision to submit the manuscript for publication.

Authors' contributions: Prafulla Shriyan was involved in the conceptualization, investigation, analysis, writingoriginal draft. Paulomi Shridhar was involved in writing- review \& editing. Onno CP van Schayck did critical review, editing of the manuscript. Giridhara R Babu supervised the study, design the methodology and involved in writing, review and editing. All authors have read and approved the manuscripts.

\section{Acknowledgements}

We sincerely thank the Department of Health and Family Welfare, Government of Karnataka (DoHFW, GoK) and the Bruhat Bengaluru Mahanagara Palike (BBMP) for permitting us to conduct the study and for providing constant sypport. We thank the hospitals under DoHFW, GoK, Superintendents, Medical Officers, doctors and all the 
departmental support staff for their support for the ongoing study. We thank Dr. Sumathi Swaminathan and her team at St. John's Research Institute for training the research assistants in anthropometry measurement. Our sincere gratitude to the Wellcome Trust, DBT India Alliance, for the support with funding and guidance. Our sincere gratitude to Dr. Suresh Shapeti and Mr. TS Ramesh for facilitating the administrative support and coordination. We also thank Deepa R and Eunice Lobo for their help in proofreading the article. We are grateful to all the research team members of MAASTHI for their support in conducting research activities in the field. We thank all participants for their effort to enroll and for continuous participation in the ongoing cohort. 


\section{References}

1. Galbally M, Lewis AJ, IJzendoorn Mv, Permezel MJHrop. The role of oxytocin in mother-infant relations: a systematic review of human studies. 2011;19(1):1-14.

2. Seth S, Lewis AJ, Saffery R, Lappas M, Galbally MJljoms. Maternal prenatal mental health and placental $11 \beta-H S D 2$ gene expression: initial findings from the mercy pregnancy and emotional wellbeing study. 2015;16(11):27482-96.

3. Lobel M, Cannella DL, Graham JE, DeVincent C, Schneider J, Meyer BAJHp. Pregnancy-specific stress, prenatal health behaviors, and birth outcomes. 2008;27(5):604.

4. Coussons-Read MEJOm. Effects of prenatal stress on pregnancy and human development: mechanisms and pathways. 2013;6(2):52-7.

5. LeWinn KZ, Stroud LR, Molnar BE, Ware JH, Koenen KC, Buka SLIjoe. Elevated maternal cortisol levels during pregnancy are associated with reduced childhood IQ. 2009;38(6):1700-10.

6. Kramer MS. Determinants of low birth weight: methodological assessment and meta-analysis. Bulletin of the world health organization. 1987;65(5):663.

7. Staneva A, Bogossian F, Pritchard M, Wittkowski A. The effects of maternal depression, anxiety, and perceived stress during pregnancy on preterm birth: A systematic review. Women and Birth. 2015;28(3):179-93.

8. Schetter C. Psychological Science on Pregnancy: Stress Processes. Biopsychosocial Models, and. 2011.

9. Seth S, Lewis AJ, Galbally MJBp, childbirth. Perinatal maternal depression and cortisol function in pregnancy and the postpartum period: a systematic literature review. 2016;16(1):1-19.

10. Brummelte S, Schmidt KL, Taves MD, Soma KK, Galea LAJDN. Elevated corticosterone levels in stomach milk, serum, and brain of male and female offspring after maternal corticosterone treatment in the rat. 2010;70(10):714-25.

11. Leinonen JA, Solantaus TS, Punamäki RLJocp, psychiatry. Parental mental health and children's adjustment: The quality of marital interaction and parenting as mediating factors. 2003;44(2):227-41.

12. Goodman SH, Rouse MH, Long Q, Ji S, Brand SRJIMHJ. Deconstructing antenatal depression: what is it that matters for neonatal behavioral functioning? 2011;32(3):339-61.

13. Lewis A, Austin E, Galbally MJJodooh, disease. Prenatal maternal mental health and fetal growth restriction: a systematic review. 2016;7(4):416-28.

14. Murray L, Arteche A, Fearon P, Halligan S, Goodyer I, Cooper PJJotAAoC, et al. Maternal postnatal depression and the development of depression in offspring up to 16 years of age. 2011;50(5):460-70.

15. Fiorentino L, Saxbe D, Alessi CA, Woods DL, Martin JLJJoGSABS, Sciences M. Diurnal cortisol and functional outcomes in post-acute rehabilitation patients. 2012;67(6):677-82.

16. Lancaster CA, Gold KJ, Flynn HA, Yoo H, Marcus SM, Davis MMJAjoo, et al. Risk factors for depressive symptoms during pregnancy: a systematic review. 2010;202(1):5-14.

17. Tsigos C, Chrousos GPJJopr. Hypothalamic-pituitary-adrenal axis, neuroendocrine factors and stress. 2002;53(4):865-71.

18. Pariante CMJJop. The glucocorticoid receptor: part of the solution or part of the problem? 2006;20(4_suppl):79-84.

19. Kammerer M, Taylor A, Glover VJAowsmh. The HPA axis and perinatal depression: a hypothesis. 2006;9(4):187-96.

20. Matthews SG, Challis JR. Regulation of the hypothalamo-pituitary-adrenocortical axis in fetal sheep. Trends in Endocrinology \& Metabolism. 1996;7(7):239-46.

21. Cottrell EC, Seckl J. Prenatal stress, glucocorticoids and the programming of adult disease. Frontiers in behavioral neuroscience. 2009;3:19.

22. Braithwaite EC, Hill J, Pickles A, Glover V, O'Donnell K, Sharp HJJodooh, et al. Associations between maternal prenatal cortisol and fetal growth are specific to infant sex: findings from the Wirral Child Health and Development Study. 2018;9(4):425-31.

23. Gilles M, Otto H, Wolf IA, Scharnholz B, Peus V, Schredl M, et al. Maternal hypothalamus-pituitary-adrenal (HPA) system activity and stress during pregnancy: Effects on gestational age and infant's anthropometric measures at birth. 2018;94:152-61.

24. Bublitz MH, Bourjeily G, D'Angelo C, Stroud LR. Maternal sleep quality and diurnal cortisol regulation over pregnancy. Behavioral sleep medicine. 2018;16(3):282-93. 
25. Smew Al, Hedman AM, Chiesa F, Ullemar V, Andolf E, Pershagen G, et al. Limited association between markers of stress during pregnancy and fetal growth in 'Born into Life', a new prospective birth cohort. Acta Paediatrica.

2018;107(6):1003-10.

26. Harville EW, Savitz DA, Dole N, Herring AH, Thorp JM. Stress questionnaires and stress biomarkers during pregnancy. Journal of women's health. 2009;18(9):1425-33.

27. Sood P, Priyadarshini S, Aich P. Estimation of psychological stress in humans: a combination of theory and practice. PloS one. 2013;8(5):e63044.

28. Babu GR, Murthy G, Deepa R, Kumar HK, Karthik M, Deshpande K, et al. Maternal antecedents of adiposity and studying the transgenerational role of hyperglycemia and insulin (MAASTHI): a prospective cohort study. BMC pregnancy and childbirth. 2016;16(1):1-9.

29. Babu GR, Murthy G, Reddy Y, Deepa R, Yamuna A, Prafulla S, et al. Small for gestational age babies and depressive symptoms of mothers during pregnancy: Results from a birth cohort in India. 2018;3.

30. Patel V, Rodrigues M, DeSouza N. Gender, poverty, and postnatal depression: a study of mothers in Goa, India. American journal of Psychiatry. 2002;159(1):43-7.

31. Babu GR, Murthy G, Reddy Y, Deepa R, Yamuna A, Prafulla S, et al. Small for gestational age babies and depressive symptoms of mothers during pregnancy: Results from a birth cohort in India. Wellcome open research. 2018;3.

32. IBM Support 2019 [updated 13th May 2019. Available from: https://www.ibm.com/support/pages/downloadingibm-spss-statistics-23.

33. Field T, Diego M. Cortisol: the culprit prenatal stress variable. International Journal of Neuroscience. 2008;118(8):1181-205.

34. Cherak SJ, Giesbrecht GF, Metcalfe A, Ronksley PE, Malebranche MEJP. The effect of gestational period on the association between maternal prenatal salivary cortisol and birth weight: A systematic review and meta-analysis. 2018;94:49-62.

35. Stewart CP, Oaks BM, Laugero KD, Ashorn U, Harjunmaa U, Kumwenda C, et al. Maternal cortisol and stress are associated with birth outcomes, but are not affected by lipid-based nutrient supplements during pregnancy: an analysis of data from a randomized controlled trial in rural Malawi. BMC pregnancy and childbirth. 2015;15(1):346.

36. Bolten MI, Wurmser H, Buske-Kirschbaum A, Papoušek M, Pirke K-M, Hellhammer D. Cortisol levels in pregnancy as a psychobiological predictor for birth weight. Archives of women's mental health. 2011;14(1):33-41.

37. Fan F, Zou Y, Zhang Y, Ma X, Zhang J, Liu C, et al. The relationship between maternal anxiety and cortisol during pregnancy and birth weight of chinese neonates. BMC pregnancy and childbirth. 2018;18(1):265.

38. Bolten MI, Wurmser H, Buske-Kirschbaum A, Papoušek M, Pirke K-M, Hellhammer DJAowsmh. Cortisol levels in pregnancy as a psychobiological predictor for birth weight. 2011;14(1):33-41.

39. Wilson $\mathrm{M}$, Thayer Z. Maternal salivary cortisone to cortisol ratio in late pregnancy: An improved method for predicting offspring birth weight. Psychoneuroendocrinology. 2017;78:10-3.

40. Goedhart G. Perinatal health epidemiology in multi-ethnic Amsterdam: Psychobiological processes. University of Amsterdam. 2010.

41. Peterson AK, M Toledo-Corral C, Chavez TA, Naya CH, Johnson M, Eckel SP, et al. Prenatal Maternal Cortisol Levels and Infant Birth Weight in a Predominately Low-Income Hispanic Cohort. International journal of environmental research and public health. 2020;17(18):6896.

42. Sloboda DM, Challis J, Moss T, Newnham JJCpd. Synthetic glucocorticoids: antenatal administration and long-term implications. 2005;11(11):1459-72.

43. Jensen E, Gallaher B, Breier B, Harding JJJoE. The effect of a chronic maternal cortisol infusion on the late-gestation fetal sheep. 2002;174(1):27-36.

44. Jensen E, Wood CE, Keller-Wood MJAJoP-R, Integrative, Physiology C. Chronic alterations in ovine maternal corticosteroid levels influence uterine blood flow and placental and fetal growth. 2005;288(1):R54-R61.

45. Gilles M, Otto H, Wolf IA, Scharnholz B, Peus V, Schredl M, et al. Maternal hypothalamus-pituitary-adrenal (HPA) system activity and stress during pregnancy: Effects on gestational age and infant's anthropometric measures at birth. Psychoneuroendocrinology. 2018;94:152-61.

46. Broekman BF, Chan YH, Chong YS, Kwek K, Sung SC, Haley CL, et al. The influence of anxiety and depressive symptoms during pregnancy on birth size. 2014;28(2):116-26. 
47. Stewart CP, Oaks BM, Laugero KD, Ashorn U, Harjunmaa U, Kumwenda C, et al. Maternal cortisol and stress are associated with birth outcomes, but are not affected by lipid-based nutrient supplements during pregnancy: an analysis of data from a randomized controlled trial in rural Malawi. BMC pregnancy and childbirth. 2015;15(1):1-12.

48. Chandra PS, Bajaj A, Desai G, Satyanarayana VA, Sharp HM, Ganjekar S, et al. Anxiety and depressive symptoms in pregnancy predict low birth weight differentially in male and female infants-findings from an urban pregnancy cohort in India. Social Psychiatry and Psychiatric Epidemiology. 2021.

49. Upadhyay AK, Srivastava SJBp, childbirth. Effect of pregnancy intention, postnatal depressive symptoms and social support on early childhood stunting: findings from India. 2016;16(1):1-14.

50. Cheng $\mathrm{CY}$, Pickler RHJS, Health. Maternal psychological well-being and salivary cortisol in late pregnancy and early post-partum. 2010;26(3):215-24.

51. Barker DJJCo, gynecology. Adult consequences of fetal growth restriction. 2006;49(2):270-83.

52. Venihaki M, Carrigan A, Dikkes P, Majzoub JAJPotNAoS. Circadian rise in maternal glucocorticoid prevents pulmonary dysplasia in fetal mice with adrenal insufficiency. 2000;97(13):7336-41. 


\section{Supplementary Files}

This is a list of supplementary files associated with this preprint. Click to download.

- Tables.pdf 